\title{
Immunoglobulin G4-related disease mimicking an epidural spinal cord tumor: case report
}

\author{
Michelle M. Williams, MD, ${ }^{1}$ Hazem Mashaly, MD, ${ }^{2,5}$ Vinay K. Puduvalli, MD, ${ }^{3}$ Ming Jin, MD, PhD, ${ }^{4}$ \\ and Ehud Mendel, MD²
}

\begin{abstract}
${ }^{1}$ College of Medicine; Departments of ${ }^{2}$ Neurological Surgery, ${ }^{3}$ Neuro-oncology, and ${ }^{4}$ Pathology, The Ohio State University James Cancer Hospital and Wexner Medical Center, Columbus, Ohio; and ${ }^{5}$ Department of Neurological Surgery, Ain Shams University, Cairo, Egypt
\end{abstract}

\begin{abstract}
The authors report a case of immunoglobulin G4-related disease (IgG4-RD) presenting as a paraspinal, epidural mass. This disease encompasses a host of autoimmune conditions that were previously thought to be separate entities. It is characterized by fibrosis, mediated by the aberrant proliferation and tissue invasion of lgG4-positive plasma cells, which can occur in any organ. As with other autoimmune conditions, it tends to be responsive to steroids and other immunosuppressants. It can rarely present as a tumefactive lesion of the central nervous system, creating the potential for misdiagnosis (given its similar radiological appearance to malignancy) and mistreatment. In 2015, a panel of experts convened to set forth guidelines for the diagnosis and treatment of $\lg G 4-R D$.

In the case presented here, the patient initially presented with pain and weakness in the left upper extremity. Initial neuroimages revealed a contrast-enhancing mass extending from C-4 to T-1, invading the epidural spinal canal, encasing the exiting nerve roots, infiltrating the paraspinal musculature, and surrounding the left vertebral artery. A PET scan confirmed the mass was hypermetabolic, but results of fine-needle aspiration and CT-guided biopsy were inconclusive. Open biopsy yielded fibrotic tissue that met the pathological criteria for IgG4-RD: Iymphoplasmacytic infiltrate, fibrosis in a storiform pattern, and obliterative phlebitis. The patient was treated with 2 doses of $4 \mathrm{mg}$ of dexamethasone (Decadron) and then $50 \mathrm{mg}$ of prednisone per day. Within 2 weeks, the mass was radiologically shown to have drastically decreased in size. The prednisone dose was decreased to $40 \mathrm{mg}$ per day, and $100 \mathrm{mg}$ of azathioprine per day was added. The patient continued to improve and the mass continued to decrease over the next 6 months. Currently, she has been weaned from all steroids and will be maintained on a daily dose of $100 \mathrm{mg}$ of azathioprine.
\end{abstract}

http://thejns.org/doi/abs/10.3171/2016.5.SPINE16119

KEY WORDS immunoglobulin G4; IgG4-related disease; paraspinal mass; epidural mass; spinal cord compression; autoimmune disease

I MMUNOGLOBULIN G4-related disease (IgG4-RD) is an autoimmune condition that was previously thought to be a wide variety of separate disorders as it has the ability to cause fibrosis in any organ system. Its appearance can mimic malignancy, potentially resulting in misdiagnosis and improper treatment. The diagnosis of IgG4-RD is made on the basis of histopathological appearance, requiring the presence of at least 2 of the following 3 criteria: lymphoplasmacytic infiltrate, fibrosis in a storiform pattern, and obliterative phlebitis. ${ }^{7}$ Because it is a relatively newly described entity, the pathophysiology behind IgG4-related fibrosis is poorly understood. Given that lesions caused by IgG4-RD are almost always responsive to steroid treatment, physicians must keep the condi- tion in mind when evaluating a possibly malignant lesion. Though there are case reports of IgG4-RD in every area of the body, $1,4,6,7,9,13-14,16,18,21-23,26$ involvement of the central nervous system (CNS) is quite rare. Here, we present a case of IgG4-RD manifesting as a cervical paraspinal mass that responded well to steroid treatment.

\section{Case Report}

History and Physical Examination

This 46-year-old female had a history of anterior cervical discectomy and fusion (ACDF) at the C6-7 level 4 years prior to presentation, fibromyalgia, and allergic contact dermatitis to poison ivy. She presented to the outpatient clinic with a 4-month history of worsening neck pain

ABBREVIATIONS ACDF = anterior cervical discectomy and fusion; CNS = central nervous system; IgG4-RD = immunoglobulin G4-related disease; IHC = immunohistochemical. 

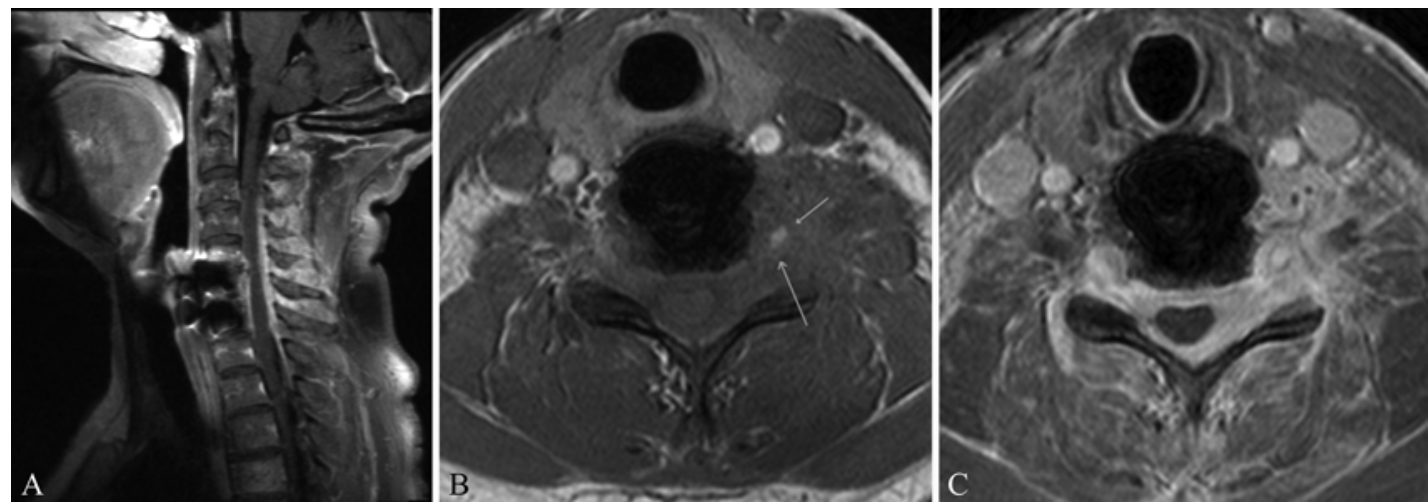

FIG. 1. Sagittal postcontrast (A) and axial precontrast (B) and postcontrast (C) T1-weighted MR images demonstrating a left-sided paraspinal mass infiltrating the spinal canal. Arrows indicate involvement of the left vertebral artery. Postoperative changes from a prior C6-7 ACDF are visible on the sagittal image.

associated with paresthesia and progressive weakness in both arms, mostly on the left side. She had been evaluated at an outside hospital with MRI of the cervical spine, which revealed a soft-tissue paraspinal mass. Additionally, she had a core needle biopsy that was inconclusive. On physical examination, her motor power was Medical Research Council (MRC) Grade 0/5 in the left deltoid and biceps muscles and Grade 2/5 in the left triceps and extrinsic hand muscles. In the right arm she was Grade $4 / 5$ in the deltoid muscle and was otherwise Grade 5/5. She had Grade $5 / 5$ motor power in both lower extremities. There was decreased light touch and pinprick sensations in the C-5, C-6, C-7, and T-1 dermatomal distributions on her left side. A positive Hoffman's sign was also present.

\section{Radiological Findings}

Magnetic resonance imaging of the cervical spine without and with contrast (Fig. 1) revealed a contrastenhancing, infiltrating, left paraspinal and epidural mass extending from $\mathrm{C}-4$ to T-1. The mass invaded the adjacent musculature and encased the left vertebral artery with circumferential involvement of the epidural space, compression of the spinal cord, and encasement of the exiting nerve roots. Of note, the mass had increased in size when compared with the MRI studies obtained 2 months earlier. Short tau inversion recovery (STIR) sequences were obtained as well but did not yield any additional diagnostic information. A PET scan showed that the mass was hypermetabolic, with no other areas concerning for malignancy.

\section{Biopsy and Results}

At this point a CT-guided biopsy as well as a fine-needle aspiration was done. Results for both were nondiagnostic, so an open biopsy of the mass was planned. The procedure was done with the patient supine, after inducing general anesthesia and with the aid of neuromonitoring. A left-sided anterior cervical approach via a transverse skin incision was performed, and blunt dissection revealed abnormal-appearing firm tissue that was poorly defined with no discernible borders along the paraspinal area and longus coli muscle. Tissue biopsy was then sent for frozen section, which came back as fibrous tissue with no evidence of malignancy. Several tissue samples were then sent for permanent pathological examination as well as culture. Closure was done in the usual sterile fashion.

According to pathological analysis, the tissue had undergone dense fibrosis, and lymphoplasmacytic inflammation was noted, as was possible focal obliterative phlebitis (Fig. 2). Malignant cells were notably absent. Immunoglobulin G4 immunohistochemical (IHC) staining was sent, and the patient was started on $4 \mathrm{mg}$ of dexamethasone (Decadron). The IgG4 IHC results revealed scattered IgG4-positive plasma cells, up to 10/hpf. Her initial IgG4 serum level before starting dexamethasone was $0.38 \mathrm{~g} / \mathrm{L}$.
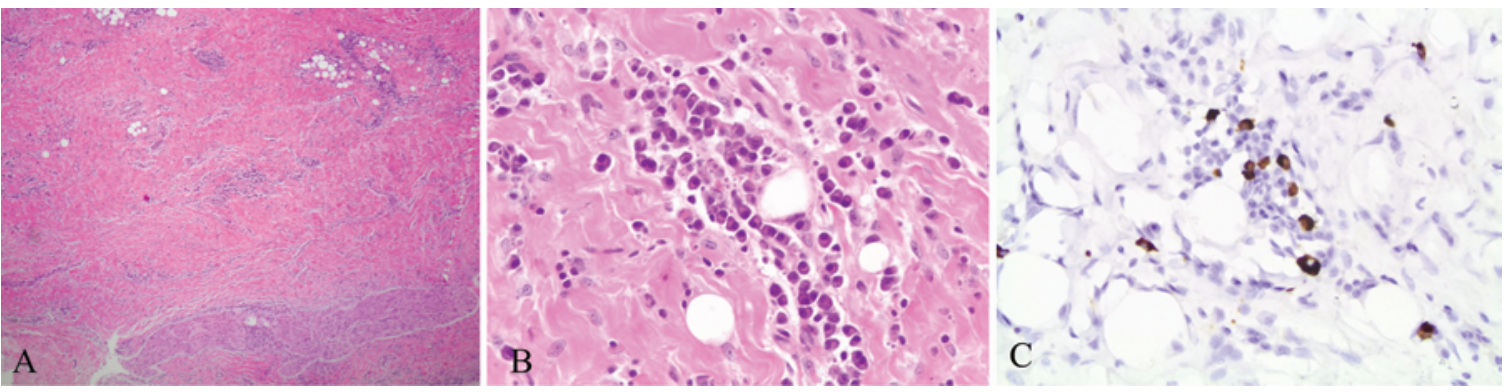

FIG. 2. Fibrosis and scattered lymphoid aggregates are observable (A). There are also focal areas of plasma cell clusters (B), some of which are positive for lgG4 (C). H \& E (A and B), original magnification $\times 4(A)$ and $\times 40(B$ and $C)$. 


\section{Treatment and Clinical Course}

The patient received 2 doses of $4 \mathrm{mg}$ of dexamethasone while under observation in the hospital and awaiting the IgG4 IHC results. Almost immediately, she reported subjective functional improvement in her weakness and pain. She was subsequently discharged on $50 \mathrm{mg}$ of prednisone per day. An MRI study performed after 2 weeks of treatment showed that the mass had decreased in size (Fig. 3). Serum levels of IgG4 at this point were $0.24 \mathrm{~g} / \mathrm{L}$, a decrease from the initial measurement of $0.38 \mathrm{~g} / \mathrm{L}$. Her steroid dose was decreased to $40 \mathrm{mg}$ of daily prednisone, and $100 \mathrm{mg}$ of daily azathioprine was added.

Three months after her initial presentation to our hospital, she reported a continued decrease in neuropathic pain. Examination of her left upper extremity revealed a motor power of Grade 3/5 in the deltoid muscle, Grade 3/5 in the triceps muscle, and Grade 4/5 in the biceps and intrinsic hand muscles. Her right upper extremity was Grade 5/5 throughout. At her final follow-up visit, 6 months after her initial presentation, her examination revealed Grade 4/5 motor power in the left deltoid, biceps, and triceps muscles and Grade 5/5 in the intrinsic hand muscles. Magnetic resonance imaging revealed stable resolution of the paraspinal mass (Fig. 4). She will continue to follow up with the rheumatology department, who plans to continue her on $100 \mathrm{mg}$ of azathioprine daily.

\section{Discussion}

Immunoglobulin G4-related disease is an autoimmune disorder that can mimic malignancy. It is an inflammatory disease that has the ability to cause fibrosis in any organ system. The pathophysiology of IgG4-RD is not completely understood at this time. It is thought that chronic exposure to antigens, pathogens, or even chronic tissue damage may preferentially upregulate differentiation into IgG4 antibody-secreting plasma cells in an effort to dampen the immune response; this aberrant repair process may then result in fibrosis, autoreactivity, and continued unnecessary production of IgG4. ${ }^{15}$ IgG4-RD often affects multiple organ systems, most typically causing sialadenitis, pancreatitis, and/or lymphadenopathy. ${ }^{6,16,26}$ Common findings in IgG4-RD include an elevated serum IgG4 level (with a ratio of $\mathrm{IgG} 4 / \mathrm{IgG}$ greater than $40 \%$ ), infiltration of organs with IgG4-positive plasma cells, and fibrosis of the affected organs. ${ }^{6,7,16}$ In the presented case, the patient's IgG4 IHC staining was on the lower end of the results reported in other cases affecting the CNS, at 10 cells/hpf. However, given her overall clinical picture (negative workup for malignancy, histopathological results consistent with IgG4-RD, positive IgG4 IHC staining, and an excellent response to steroids), we are confident in the diagnosis of IgG4-RD.

\section{Tumefactive Appearance}

Estimates of the prevalence of tumefactive lesions (rather than diffuse fibrosis) in IgG4-RD vary: a study of newly diagnosed patients in China reported an $8.5 \%$ prevalence, ${ }^{16}$ while a similar study in Japan reported a $2.3 \%$ prevalence. ${ }^{13}$ There are case reports in which masses that have the radiological appearance of cancerous tumors
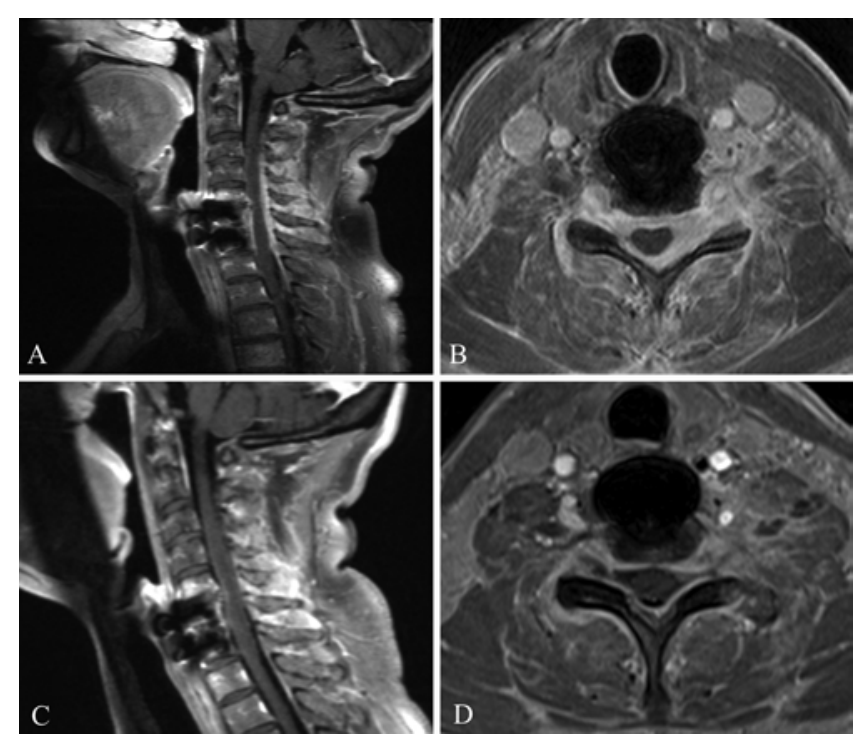

FIG. 3. Sagittal (A) and axial (B) postcontrast T1-weighted MR images obtained at initial presentation (prior to initiation of treatment), and sagittal (C) and axial (D) postcontrast T1-weighted MR images obtained 2 weeks postinitiation of steroid treatment. The enhancing paraspinal mass infiltrating the spinal canal in the initial pretreatment images is seen to decrease in size in the posttreatment images.

are subsequently biopsied and demonstrate no malignant cells, instead showing the histopathological characteristics consistent with IgG4-RD. ${ }^{20,26}$ These lymphoplasmacytic lesions tend to affect solid organs such as the pancreas, thyroid, lung, and kidney. ${ }^{1,13,26}$

\section{Central Nervous System Involvement}

One very rarely reported site of IgG4-RD lesions is the CNS. Only a handful of case reports describe this phenomenon, including dural ${ }^{2,12,17,25}$ and epidural ${ }^{3}$ masses in the cervical and thoracic spine, intracranial masses, ${ }^{5,25}$ and a mass at the L-5 nerve root. ${ }^{27}$ Additionally, it has been shown that some cases previously described as idiopathic hypertrophic pachymeningitis are in fact attributable to IgG4-RD. ${ }^{17,27}$ In these cases, there was no definitive association with pathology in other organ systems; some cases reported prior or subsequent involvement of the salivary glands ${ }^{2}$ or kidneys,${ }^{25}$ whereas other cases reported no other organ involvement at the time of publication. In all cases, diagnosis was based on the histopathological features of the mass, showing the typical fibrosis and IgG4 cells. Most reported a favorable response to steroid treatment, $, 3,17,25$ except in one patient whose weakness progressed despite being on intravenous pulse steroids and who failed to recover even after resection of the mass. ${ }^{12}$ In one case, the epidural mass recurred 2 months after being resected, but the patient had not been receiving steroids; prednisolone was started after the second resection, and the mass regressed to the point where the patient was no longer symptomatic. ${ }^{3}$

\section{Diagnosis}

Currently, the literature supports specific pathological features for diagnosis, at least 2 of the following 3 factors: 

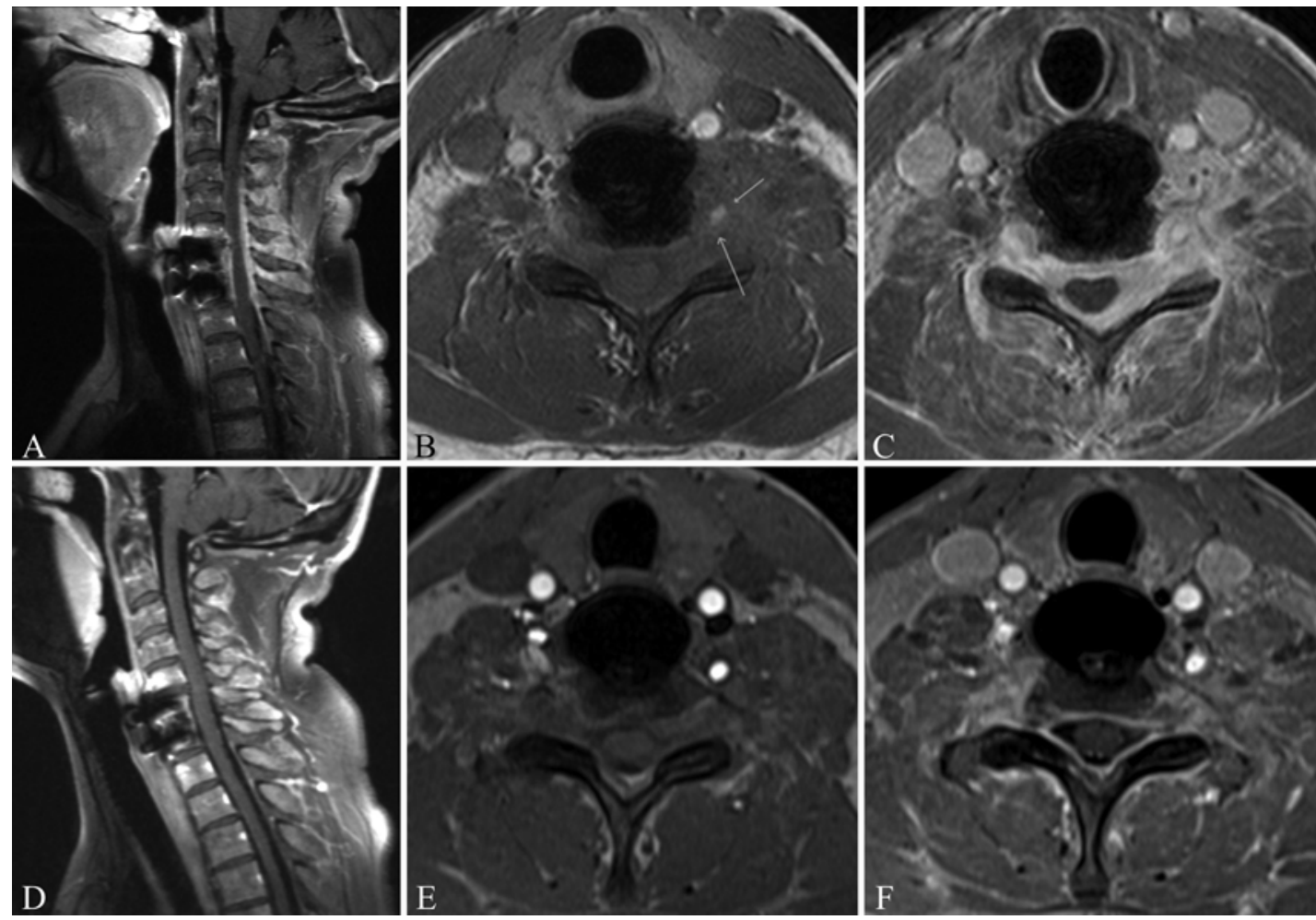

FIG. 4. Sagittal postcontrast (A) and axial precontrast (B) and postcontrast (C) T1-weighted MR images obtained at the initial presentation (prior to initiation of treatment). Sagittal postcontrast (D) and axial precontrast (E) and postcontrast (F) T1-weighted MR images obtained 6 months after initiation of steroid therapy. The enhancing paraspinal and epidural mass seen in the initial images $(A-C)$ shows stable improvement after 6 months of treatment (D-F). Arrows indicate involvement of the left vertebral artery.

dense lymphoplasmacytic infiltrate, fibrosis in a storiform pattern, and obliterative phlebitis.? In our presented case, the tissue obtained by open biopsy fit all 3 major criteria. These findings must be interpreted within the clinical context as they are relatively nonspecific when viewed alone. Additionally, these criteria can be met in some patients with isolated asymptomatic lymphadenopathy and in such cases do not warrant the diagnosis of IgG4-RD or its treatment. ${ }^{19}$ Though IgG4 serum elevation was initially investigated as a possible disease marker in the literature, it must be interpreted cautiously. Elevations ranging anywhere from 5 to 50 times the normal range have been reported (normal range $0.05-1.4 \mathrm{~g} / \mathrm{L}$ ). ${ }^{15}$ However, one study of clinical characteristics in patients with IgG4-RD demonstrated normal IgG4 serum levels in 50\% of patients with biopsy-confirmed disease. ${ }^{28}$ In the present case, the patient's serum IgG4 was not elevated. Neither did she have any other known organ involvement at the time of diagnosis. Nevertheless, it is important to consider IgG4-RD as a potentially systemic disease that carries the possibility of future manifestations in other organ systems.

Physicians should also keep in mind that other conditions can present with an elevated IgG4 serum level, such as granulomatosis with polyangiitis. ${ }^{24}$ Thus, an elevated IgG4 serum level has not been proven to be sensitive or specific for IgG4-RD. There is some evidence that the number of circulating bloodstream plasmablasts (immature plasma cells capable of secreting large quantities of antibodies) is a more reliable disease marker. ${ }^{29}$ Though this can be determined by flow cytometry, there is currently no established cutoff to make a definitive diagnosis, and thus the circulating plasmablast number is not included in the international 2015 consensus on diagnosis and treatment guidelines. ${ }^{11}$

\section{Treatment}

When suspicion for IgG4-RD is high, patients can be started on a trial of glucocorticoids, which are considered first-line treatment for inducing remission. ${ }^{5}$ Unfortunately, sustained remission may not be accomplished with glucocorticoids, with one study reporting a failure rate of $77 \% .28$ Some studies have demonstrated a response to other immunosuppressants or rituximab, especially when glucocorticoids have failed to maintain remission. ${ }^{8,10,11,16}$ In the present case, the mass rapidly responded to an initial treatment regimen of $50 \mathrm{mg}$ of daily prednisone, which was completely weaned eventually. A good response to glucocorticoids, as measured by a decrease in the size of the lesion and sometimes even a resolution of the lesion entirely, favors the diagnosis of IgG4-RD. The long-term management plan in our patient is the continuation of 100 $\mathrm{mg}$ of daily azathioprine and follow-up with rheumatology. Follow-up recommendations include periodic imaging and measurement of serum IgG4 levels as some patients will require maintenance glucocorticoid therapy or additional immunosuppresants. ${ }^{11}$ 


\section{Conclusions}

Immunoglobulin G4-related disease is a diagnosis for clinicians to bear in mind when working up a mass in any part of the body because early suspicion based on the typical histopathological features could encourage a trial of glucocorticoids and spare the patient unnecessary surgery or cancer treatments. As there are very few reported cases of IgG4-RD manifesting as a spinal cord mass, it is difficult to recognize and treat. As in all cases of suspected IgG4-RD, a biopsy is the best diagnostic option to differentiate this disease from malignancy, especially lymphoma. Elevated IgG4 serum levels are not required for diagnosis and are considered of secondary importance to the histopathological findings. Glucocorticoids are recommended as first-line therapy but can fail to sustain remission, requiring additional or the alternate use of other immunosuppressants.

\section{References}

1. Beyer G, Schwaiger T, Lerch MM, Mayerle J: IgG4-related disease: a new kid on the block or an old acquaintance? United European Gastroenterol J 2:165-172, 2014

2. Chan SK, Cheuk W, Chan KT, Chan JKC: IgG4-related sclerosing pachymeningitis: a previously unrecognized form of central nervous system involvement in IgG4-related sclerosing disease. Am J Surg Pathol 33:1249-1252, 2009

3. Choi SH, Lee SH, Khang SK, Jeon SR: IgG4-related sclerosing pachymeningitis causing spinal cord compression. Neurology 75:1388-1390, 2010

4. Chougule A, Bal A, Das A, Singh G: IgG4 related sclerosing mastitis: expanding the morphological spectrum of IgG4 related diseases. Pathology 47:27-33, 2015

5. Della-Torre E, Passerini G, Furlan R, Roveri L, Chieffo R, Anzalone N, et al: Cerebrospinal fluid analysis in immunoglobulin G4-related hypertrophic pachymeningitis. J Rheumatol 40:1927-1929, 2013

6. Deshpande V: IgG4-related disease of the gastrointestinal tract: a 21st century chameleon. Arch Pathol Lab Med 139:742-749, 2015

7. Deshpande V, Zen Y, Chan JK, Yi EE, Sato Y, Yoshino T, et al: Consensus statement on the pathology of IgG4-related disease. Mod Pathol 25:1181-1192, 2012

8. Gillispie MC, Thomas RD, Hennon TR: Successful treatment of IgG-4 related sclerosing disease with rituximab: a novel case report. Clin Exp Rheumatol 33:549-550, 2015

9. Hasosah MY, Satti MB, Yousef YA, Alzahrani DM, Almutairi SA, Alsahafi AF, et al: IgG4-related sclerosing mesenteritis in a 7-year-old Saudi girl. Saudi J Gastroenterol 20:385-388, 2014

10. Iwasaki S, Kamisawa T, Koizumi S, Chiba K, Tabata T, Kuruma $S$, et al: Assessment in steroid trial for IgG4-related sclerosing cholangitis. Adv Med Sci 60:211-215, 2015

11. Khosroshahi A, Wallace ZS, Crowe JL, Akamizu T, Azumi A, Carruthers MN, et al: International consensus guidance statement on the management and treatment of IgG4-related disease. Arthritis Rheumatol 67:1688-1699, 2015

12. Kim SH, Kang Y, Oh SH, Paik S, Kim JS: Paraplegia in a patient with IgG4-related sclerosing disease: a case report. Ann Rehabil Med 38:856-860, 2014

13. Koizumi S, Kamisawa T, Kuruma S, Tabata T, Chiba K, Iwasaki S, et al: Organ correlation in IgG4-related diseases. J Korean Med Sci 30:743-748, 2015

14. Lee CS, Harocopos GJ, Kraus CL, Lee AY, Van Stavern GP, Couch SM, et al: IgG4-associated orbital and ocular inflammation. J Ophthalmic Inflamm Infect 5:15, 2015
15. Lighaam LC, Aalberse RC, Rispens T: IgG4-related fibrotic diseases from an immunological perspective: regulators out of control? Clinical article. Int J of Rheumatol 2012:789164, 2012

16. Lin W, Lu S, Chen H, Wu Q, Fei Y, Li M, et al: Clinical characteristics of immunoglobulin G4-related disease: a prospective study of 118 Chinese patients. Rheumatology (Oxford) 54:1982-1990, 2015

17. Lindstrom KM, Cousar JB, Lopes MBS: IgG4-related meningeal disease: clinico-pathological features and proposal for diagnostic criteria. Acta Neuropathol 120:765-776, 2010

18. Mahajan VS, Mattoo H, Deshpande V, Pillai SS, Stone JH: IgG4-related disease. Annu Rev Pathol 9:315-347, 2014

19. Martinez LL, Friedländer E, van der Laak JA, Hebeda KM: Abundance of IgG4+ plasma cells in isolated reactive lymphadenopathy is no indication of IgG4-related disease. Am J Clin Pathol 142:459-466, 2014

20. McKinnon T, Randazzo WT, Kim BD, Biddinger P, Forseen $\mathrm{S}$ : IgG4-related disease presenting as a solitary neck mass. J Radiol Case Rep 9:1-8, 2015

21. Nakazawa T, Ikeda Y, Kawaguchi Y, Kitagawa H, Takada H, Takeda Y, et al: Isolated intrapancreatic IgG4-related sclerosing cholangitis. World J Gastroenterol 21:1334-1343, 2015

22. Nath V, Lewin J, Subramony C, Shenoy V: IgG4-associated cholangitis. J Miss State Med Assoc 55:384-388, 2014

23. Oriot P, Amraoui A, Rousseau E, Malvaux P, Dechambre S, Delcourt A: Fibrosis of the thyroid gland caused by an IgG4related sclerosing disease: three years of follow-up. Acta Clin Belg 69:446-450, 2014

24. Perez Alamino R, Martínez C, Espinoza LR: IgG4-associated vasculitis. Curr Rheumatol Rep 15:348, 2013

25. Tajima Y, Mito Y: Cranial neuropathy because of IgG4related pachymeningitis; intracranial and spinal mass lesions. Clinical article. BMJ Case Rep 2012:bcr2012006471, 2012

26. Tanaka K, Harada H, Kayamori K, Omura K: Chronic sclerosing sialadenitis of the submandibular gland as the initial symptom of IgG4-related disease: a case report. Tohoku J Exp Med 236:193-198, 2015

27. Wallace ZS, Carruthers MN, Khosroshahi A, Carruthers R, Shinagare S, Stemmer-Rachamimov A, et al: IgG4-related disease and hypertrophic pachymeningitis. Medicine (Baltimore) 92:206-216, 2013

28. Wallace ZS, Deshpande V, Mattoo H, Mahajan VS, Kulikova M, Pillai S, et al: IgG4-related disease: clinical and laboratory features in one hundred twenty-five patients. Arthritis Rheumatol 67:2466-2475, 2015

29. Wallace ZS, Mattoo H, Carruthers M, Mahajan VS, Della Torre E, Lee H, et al: Plasmablasts as a biomarker for IgG4related disease, independent of serum IgG4 concentrations. Ann Rheum Dis 74:190-195, 2015

\section{Disclosures}

The authors report no conflict of interest concerning the materials or methods used in this study or the findings specified in this paper.

\section{Author Contributions}

Conception and design: Puduvalli, Mendel. Acquisition of data: Puduvalli, Jin, Mendel. Analysis and interpretation of data: all authors. Drafting the article: Williams, Mashaly. Critically revising the article: all authors. Reviewed submitted version of manuscript: all authors. Study supervision: Mendel.

\section{Correspondence}

Michelle M. Williams, 2409 Summit St., Columbus, OH 43202. email: michelle.williams2@osumc.edu. 